\title{
The Rest Mass of a Particle in a Field and a General Mass Equation
}

\author{
Mohamed Ahmed Hassan* \\ Department of Mathematics, Faculty of Science, Ain-Shams University, Cairo, Egypt \\ *Corresponding author: el_hmam.1113@Yahoo.com \\ Received May 26, 2014; Revised June 07, 2014; Accepted June 22, 2014
}

\begin{abstract}
Using a modified version of the mass vector model, Hassan (1997), we explain that the particle mass decreases (increases) if the particle in attractive (repulsive) field. The concepts of the free rest mass and the rest mass in a field are introduced. We conclude that the mass of a particle in a field changes according to the rule $m^{f}=m_{0}^{f} / \sqrt{1-(v / c)^{2}}$, where $m_{0}^{f}$ is the rest mass of the particle in the field. This result is consistent with the existence of the Higgs field through the space and it causes existing particles to acquire mass. The confined theory is explained. General mass equations of a particle and of a composite particle in terms of different four forces of nature are obtained. Some applications of the general mass equation of composite particle (nucleus) are presented and discussed.
\end{abstract}

Keywords: mass vector- charge vector- mass space of a force-rest mass in a field- general mass space- general mass equation

Cite This Article: Mohamed Ahmed Hassan, "The Rest Mass of a Particle in a Field and a General Mass Equation.” International Journal of Physics, vol. 2, no. 3 (2014): 86-95. doi: 10.12691/ijp-2-3-4.

\section{Introduction}

In 1997 Hassan [1] suggested a model where a one object (vector) represents the mass of the particle and its own field. He introduced the total mass of the particle concept which was defined to be $m_{t}=m+W$, where $m$ is the measured mass of the particle and $W$ is the virtual mass which is related to the field of the particle. This idea is similar, in some sense, to the idea of the electromagnetic mass [2], where the electromagnetic field contributes to the mass of charged particles. Also, the binding energy of composite particle can be considered as the part of the mass which represents the field. In the present work we try, suggesting a general definition for the charge of any force, to give a relation between the mass and the charge of the particle. To formulate the suggested definition for the charge a mathematical model for the mass of the particle with its own field is needed. This model was given by (Hassan, 1997) [1]. A modified and extended version of this model is presented in section 3. Using this model we try to answer the question: Is there a difference between the rest mass of a free particle and its rest mass in a field? Also, we try to compare between the semi-empirical Weizsäcker mass formula, [3], of nuclei and a general mass equation which is obtained by the modified mass vector model.

\section{The Charge}

Between electric charged particles there is electromagnetic force, without the electric charge the electromagnetic force between the particles does not exist. This means that, "the charge is the source of the force ". Also, between any two nucleons there is a nuclear (strong) force, and we have a question: What is the source of the nuclear force? We can, as in the case of electromagnetic force, suppose that a nuclear charge is the source of the nuclear force. Similarly, we can say that a weak charge and a gravitational charge are the sources of the weak and gravitational forces, respectively. The statement "charge is the source of the force" is not determine the nature of charge, i. e., it is not a definition of charge, and it is only represent the status. Thus, still we have the question: What is the charge for any kind of well-known four forces?

Any particle is a collection of energy. A part of this energy gives the measured mass of the particle and the other part makes the field of the particle. This field may be represents electromagnetic, strong, weak or gravitational force, or may be represents a combination of some or all of these forces. Thus, sometimes a part of energy makes electromagnetic force (field), sometimes makes strong force (nuclear force) and so on. Therefore, we can suggest that a part of energy with a certain configuration gives some kind of force, and another part of energy with another configuration gives another kind of force and so on. Then, we can define the charge of some kind of force as an (eigen) configuration of this part of energy making the field of the force. I.e., we can say that the charge is a part of energy with specific (eigen) configuration. This configuration is responsible of all properties of the considered kind of force, even the generation of the particle of the field. With this definition of the charge we can understand, not only the electric charge, but also the 
suggested concepts of nuclear, weak and gravitational charges. This generalization of charge concept may be a step to a unified field theory.

Also, we must note that there is no any definition of well-known electric charge or any other charge of any other kind of force in any literature of physics. In classical and quantum physics only described, using the concepts of the field and field particles, how a charged particle acts on the other charged particles and how any particle with strong, weak and gravitational forces acts on the other particles. But, in any case the nature of the electric charge and the nature of sources of the other forces are not defined and explained.

\section{The Mass Vector Model}

Hassan (1997) [1] defined a 4-vector of the particle mass to represent the particle with its own field, where the magnitude of the vector is related to the measured mass of the particle and its components are related to the field. The definition of this vector takes the following facts into account: (i) The equivalence relation between the mass and energy, $\mathrm{E}=\mathrm{m}$, with $\mathrm{c}=1$, where $\mathrm{c}$ is the speed of light. (ii) The mass of a complex particle, in general, is less than the sum of the masses of its constituents (triangular inequality of the vectors). (iii) The binding energy of any system of particles due to any kind of force is a part of masses of the particles. (iv) The force between the particles, according to the quantum field theory, is translated by means of the particle of the field. A modified version of this model is presented in the next sections, where the new definition of mass vectors addition is given and the mass vectors of a mass-less particle, a field, and a charge are, also, defined.

\subsection{The Mass Vector and the Definition of Charge}

In the mass vector model [1], the mass vector of a particle having some kind of force was defined as a fourvector

$$
\mathbf{m}=\left(m^{0}, m^{1}, m^{2}, m^{3}\right)
$$

such that "the magnitude of the mass vector $=\sqrt{m}$ ", where $m$ is the measured mass of the particle. The component $m^{0}=\sqrt{m_{t}}$, where $m_{t}$ is the total mass of the particle, i.e., the sum of the measured mass of the particle $m$ and its virtual mass $W$. The virtual mass $W$ is the used energy to construct the field of the considered kind of force of the particle. Thus, $m_{t}=m+W$. The other three components $m^{1}, m^{2}$ and $m^{3}$ are related to the particle field such that

$$
W=\left(m^{1}\right)^{2}+\left(m^{2}\right)^{2}+\left(m^{3}\right)^{2}
$$

We usually write,

$$
\mathbf{W}=\left(m^{1}, m^{2}, m^{3}\right)
$$

Consequently,

$$
\mathbf{m}=\left(\sqrt{m_{t}}, \mathbf{W}\right)
$$

In this representation, the vector $\mathbf{W}$ can be considered as the source of the force of the particle. This vector with certain values for the components $m^{1}, m^{2}$ and $m^{3}$ in three specific directions inside the particle gives a specific energy configuration. This configuration corresponds to the considered kind of force of the particle. Different configuration (with another three specific directions inside the particle) gives different kind of force. Thus, a configuration is defined by three specific directions inside the particle. We will assume that these directions are fixed with respect to the particle and can be considered as axes of some kind of symmetry of the particle. Different sets of values of the components $m^{1}, m^{2}$ and $m^{3}$ with respect to the same axes give fields of the same kind of force, but for different particles. For example, the representations of the same electric charges in $e^{+}, \pi^{+}$and $p$ are different. Therefore, we can assume that these particles have different sets of values of components $m^{1}, m^{2}$ and $m^{3}$. But, all these particles have the same electromagnetic force. According to the quark model, we can expect $\mathbf{W}$ with one nonzero component for $e^{+}$, with two nonzero components for $\pi^{+}$and with three nonzero components for $p$. Now, we can say that, for any particle having $N$ kinds of force we have $N$ different mass vectors corresponding to these different kinds of force. All these vectors have the same magnitude $m$ with respect to the same time-space reference where the particle is considered, but with different configurations for $\mathbf{W}$. We have only, in present time, four energy configurations corresponding to four well-known kinds of force: strong, electromagnetic, gravitational and weak.

Thus, we can define a charge of some kind of force as an energy configuration representing by a vector $\mathbf{W}$ with respect to a set of three specific axes inside the particle. These axes will be called "charge axes" and are considered a principle property of all particles having the same kind of force. Since for any particle the largest number of electric charge fractions is three, we can consider that the assumption of three charge axes may be correct, and then the four dimensions of the mass vector can be accepted. I.e., when the mass is represented by a 4vector $\mathbf{m}=\left(\sqrt{m_{t}}, \mathbf{W}\right)$, the charge can be represented by a 3-vector $\mathbf{W}$.

The magnetic field of a magnet represents a macroscopic classical example for a field which is coming from directed masses, i.e., if the molecules of the iron are directed in some certain direction we have a field of the magnetic force.

Now, the question about the definition of charge is reduced to the question about the charge axes, values of components in the directions of these axes and the relation between these values and, for example, the values of classical charge in the case of electromagnetic force. All these concepts represent open questions in this model.

For any particle, we have now four principal charge vectors $\mathbf{W}_{s}, \mathbf{W}_{e}, \mathbf{W}_{g}$ and $\mathbf{W}_{w}$ corresponding to strong, 
electromagnetic, gravitational and weak forces, respectively. Each of these vectors is considered with respect to different sets of charge axes. This means that the vector $\mathbf{W}_{i}$ belongs to different reference frame for different values of $i$. Thus, we have four different reference frames each of which is related to a certain kind of force. Then, the mass vector $\mathbf{m}=\left(\sqrt{m_{t}}, \mathbf{W}\right)$ belongs to one and only one four-dimensional space for a certain kind of force. We will call this four dimensional space by the "mass space".

We must note that in any "mass space" of a certain force the other forces are neglected. This means that the total mass of a particle $m_{t}=m+W$ has an approximated value, but, the measured mass $m$ is the same in all mass spaces.

According to this representation of the total energy of the particle and the charge definition, we can imagine a closed system representing by $\mathbf{W}_{i}$ which is associated to the set of charge axes of a certain kind of force. Closed system means that this system does not interact with any other system $\mathbf{W}_{j}, j \neq i$ of the other kind of force. This is a logical situation. For example, the electron interacts with any electromagnetic field, but, it does not interact with the strong field of the nucleus.

\subsection{The Mass Spaces}

The elements of a mass space are the mass vectors of all different particles having the same definite kind of force. I.e., we have different four mass spaces $M_{s}, M_{e}$, $M_{g}$ and $M_{w}$ corresponding to the four kinds of force and in each one the other forces are neglected. In any mass space, each particle has one and only one mass vector in the space. Therefore, any particle has at all four mass vectors $\mathbf{m}_{s}, \mathbf{m}_{e}, \mathbf{m}_{g}$ and $\mathbf{m}_{w}$ corresponding to strong, electromagnetic, gravitational, and weak force, respectively. These vectors belong to different mass spaces and each of them belongs to one and only one mass space. The magnitudes of all mass vectors of the same particle have the same value and are obeyed to the special theory of relativity in the space-time reference frame, but the total mass of the particle in each space takes different values. It is clear that each of these mass spaces corresponds to some classical partition of physics.

\subsection{Addition of Two Mass Vectors}

In any mass space $M_{\alpha}$ the addition of two mass vectors $\quad \mathbf{m}_{1}=\left(m_{1}^{0}, m_{1}^{1}, m_{1}^{2}, m_{1}^{3}\right)$ and

$\mathbf{m}_{2}=\left(m_{2}^{0}, m_{2}^{1}, m_{2}^{2}, m_{2}^{3}\right)$ of any two particles 1 and 2 is defined such that the physical fusion of these two particles corresponds to the sum of the two corresponding mass vectors. Hassan (1997) [1] suggested the following definition for the addition of two mass vectors, we will call it the first definition.

The first definition: The mass vector of the new composite particle $\mathbf{m}$ is given by [1] $\mathbf{m}=\mathbf{m}_{1}+\mathbf{m}_{2}=\left(m_{1}^{0}+m_{2}^{0}, m_{1}^{1}+m_{2}^{1}, m_{1}^{2}+m_{2}^{2}, m_{1}^{3}+m_{2}^{3}\right)$.

This definition leads to some unusual results. For example, for the total mass of the composite particle $\mathbf{m}=\mathbf{m}_{1}+\mathbf{m}_{2}$ we have

$$
\begin{aligned}
& m_{t}=\left(m^{0}\right)^{2}=\left(\sqrt{m_{1 t}}+\sqrt{m_{2 t}}\right)^{2} \\
& =m_{1 t}+m_{2 t}+2 \sqrt{m_{1 t}} \sqrt{m_{2 t}}>m_{1 t}+m_{2 t} .
\end{aligned}
$$

This might be due to what we can call the resonance of the composition of the two total masses (energies) together. Therefore, in this work we try to suggest another definition. Using this second definition we can give a direct application of the model and obtain some information about the particle-particle interaction.

The second definition: The mass vector of the new composite particle $\mathbf{m}$ is given by

$$
\begin{aligned}
& \mathbf{m}=\mathbf{m}_{1}+\mathbf{m}_{2}=\left(\sqrt{\left(m_{1}^{0}\right)^{2}+\left(m_{2}^{0}\right)^{2}},\right. \\
& \left.m_{1}^{1}+m_{2}^{1}, m_{1}^{2}+m_{2}^{2}, m_{1}^{3}+m_{2}^{3}\right) .
\end{aligned}
$$

In the general case of $n$-constituent particle of mass vector ${ }^{\mathbf{m}}$ we have

$$
\mathbf{m}=\sum_{i=1}^{n} \mathbf{m}_{i}=\left(\left(\sum_{i=1}^{n}\left(m_{i}^{0}\right)^{2}\right)^{1 / 2}, \sum_{i=1}^{n} m_{i}^{1}, \sum_{i=1}^{n} m_{i}^{2}, \sum_{i=1}^{n} m_{i}^{3}\right)
$$

In terms of the charge vector, equation (7) can be written as

$$
\mathbf{m}=\sum_{i=1}^{n} \mathbf{m}_{i}=\left(\sqrt{m_{t}}, \mathbf{W}\right)=\left(\left(\sum_{i=1}^{n} \mathbf{m}_{i t}\right)^{1 / 2}, \sum_{i=1}^{n} \mathbf{W}_{i}\right),
$$

i.e., from equation (8), we have $m_{t}=\sum_{i=1}^{n} m_{i t}$.

Form this definitions, it is clear that, if $\mathbf{m}, \mathbf{m}_{1}, \mathbf{m}_{2}$, and $\mathbf{m}_{3}$ are any four mass vectors in a given mass space we have

$$
\begin{gathered}
\mathbf{m}_{1}+\mathbf{m}_{2}=\mathbf{m}_{2}+\mathbf{m}_{1} \\
\mathbf{m}_{1}+\left(\mathbf{m}_{2}+\mathbf{m}_{3}\right)=\left(\mathbf{m}_{1}+\mathbf{m}_{2}\right)+\mathbf{m}_{3}
\end{gathered}
$$

$$
\mathbf{m}+\mathbf{o}=\mathbf{o}+\mathbf{m}=\mathbf{m}
$$

where $\mathbf{o}=(0,0,0,0)$ is the mass vector of the empty space (vacuum).

\subsection{The Inner Product of Two Mass Vectors}

In any mass space the inner product of two mass vectors

$$
\mathbf{m}_{1}=\left(m_{1}^{0}, m_{1}^{1}, m_{1}^{2}, m_{1}^{3}\right)
$$

and $\mathbf{m}_{2}=\left(m_{2}^{0}, m_{2}^{1}, m_{2}^{2}, m_{2}^{3}\right)$ of any two particles denoted by $\mathbf{m}_{1} \circ \mathbf{m}_{2}$ and is defined as

$$
\mathbf{m} \circ \mathbf{m}=m_{1}^{0} m_{2}^{0}-m_{1}^{1} m_{2}^{1}-m_{1}^{2} m_{2}^{2}-m_{1}^{3} m_{2}^{3}
$$

Thus, for any particle of mass vector $\mathbf{m}=\left(m^{0}, m^{1}, m^{2}, m^{3}\right)$, we have

$$
\mathbf{m} \circ \mathbf{m}=\left(m^{0}\right)^{2}-\left(m^{1}\right)^{2}-\left(m^{2}\right)^{2}-\left(m^{3}\right)^{2}=m_{t}-W=m(13)
$$


It is clear that $\mathbf{m}_{1} \circ \mathbf{m}_{2}=\mathbf{m}_{2} \circ \mathbf{m}_{1}$. In terms of the charge vector, the inner product of two vectors, Eq. (12 ), can be written as

$$
\mathbf{m}_{1} \circ \mathbf{m}_{2}=\sqrt{m_{1 t}} \sqrt{m_{2 t}}-\mathbf{W}_{1} \bullet \mathbf{W}_{2}
$$

where the black circle is used to represent the inner product of the usual three-dimensional vectors

$$
\mathbf{W}_{1} \bullet \mathbf{W}_{2}=m_{1}^{1} m_{2}^{1}+m_{1}^{2} m_{2}^{2}+m_{1}^{3} m_{2}^{3}
$$

From the second definition of addition of two mass vectors, $\mathbf{m}=\mathbf{m}_{1}+\mathbf{m}_{2}$, we have

$$
\begin{aligned}
m= & \left(\sqrt{m_{1 t}+m_{2 t}}\right)^{2} \\
& -\left(W_{1}+W_{2}+2 \mathbf{W}_{1} \bullet \mathbf{W}_{2}\right) \\
& =m_{1 t}-W_{1}+m_{2 t}-W_{2}-2 \mathbf{W}_{1} \bullet \mathbf{W}_{2} \\
& =m_{1}+m_{2}-2 \mathbf{W}_{1} \bullet \mathbf{W}_{2},
\end{aligned}
$$

where we consider $\mathbf{W}_{1} \bullet \mathbf{W}_{2}=\mathbf{W}_{2} \bullet \mathbf{W}_{1}$. May be convenient to write

$$
\mathbf{W}_{1} \bullet \mathbf{W}_{2}=\lambda_{12} \sqrt{W_{1}} \sqrt{W_{2}}
$$

where $\lambda_{12}$ is an interaction parameter of the two particles. Of course, $\lambda_{12}$ is not $\cos \theta$ of any space angle $\theta$. It is a parameter which is related to the interaction between the two particles. Equation (16) becomes

$$
m=m_{1}+m_{2}-2 \lambda_{12} \sqrt{W_{1}} \sqrt{W_{2}}
$$

We have three cases for the values of $\lambda_{12}$. The first case is $\lambda_{12}=0$. In this case the two particles are independent, i.e., each of them outside the field of the other. In the second case $\lambda_{12}<0$. This case represents a new composite particle with the mass $m>m_{1}+m_{2}$ as in the case of $\rho$ - and ${ }^{\Delta}$-particle (resonance), which explain the confined theory, Griffiths (1987) [4]. In the third case where $\lambda_{12}>0$, we have a new composite particle with the mass $m<m_{1}+m_{2}$, which is considered as the usual case of nuclei and the most of particles.

\subsection{Lorentz Transformations Between Two Mass Spaces}

Each mass space is characterized by a specific set of charge axes, i.e., the reference axes in any two different mass spaces are, in general, different. At the same time, the magnitudes of the four mass vectors, $\mathbf{m}_{s}, \mathbf{m}_{e}, \mathbf{m}_{g}$ and $\mathbf{m}_{w}$ of the same particle in different mass spaces $M_{s}, M_{e}, M_{g}$ and $M_{w}$ have the same value. Since in any Minkovski space the magnitude of any vector must be invariant under Lorentz transformations, Hassan (1997) [1] suggested that the mass vectors are obeyed to the following Lorentz transformations

$$
\begin{aligned}
& m^{\prime 0}=\frac{m^{0}+\alpha m^{1}}{\sqrt{1-\alpha^{2}}}, m^{\prime}=\frac{m^{1}+\alpha m^{0}}{\sqrt{1-\alpha^{2}}} \\
& m^{\prime 2}=m^{2}, m^{\prime 3}=m^{3}
\end{aligned}
$$

where ${ }^{\alpha}$ is a transformation parameter. This parameter $\alpha$ cannot take the same meaning of $v / c$, where $v$ is the velocity of one system with respect to the other. Taking $\alpha=v / c$, we have $m=m_{0}$ while the correct relation is $m=m_{0} / \sqrt{1-\alpha^{2}}, m_{0}$ is the rest mass of the particle. At the same time, for any mass vector $\mathbf{m}=\left(m^{0}, m^{1}, m^{2}, m^{3}\right)$, from (13) and (19), we have $m_{t}-W=m_{t}^{\prime}-W^{\prime}$, i.e., $m=m^{\prime}$. This representation of the vector transformation is similar to a special Lorentz transformation in the theory of relativity. The difference between the two cases is the sense of the parameter $\alpha$ of the transformation.

In fact, we believe that the transformation from the mass space of one kind of force to another mass space of another kind of force is not simply as above. It might be more realistic if we suggest that the mass vectors obey to rotational Lorentz transformations. This does not mean that the first force transform to the other force, but, only means a mathematical transformation from a fixed frame to the other fixed frame. In the present work we are not concerned with the details of these transformations.

\subsection{The Norm of the Mass Vector}

For a massless particle, the measured mass of the particle equals zero. But, this particle represents a quant of energy. Therefore, the total mass of the particle is not zero. It gives some field. Thus, according to our definition of the mass vector $\mathbf{m}$, the magnitude of the mass vector $\mathbf{m}$ may be equals zero even with $\mathbf{m} \neq 0$ (since $m^{0}=\sqrt{m_{t}} \neq 0$ except in the case of the vacuum). Therefore, the axiom of mathematical norm, $\|\mathbf{a}\|=0$ if and only if $\mathbf{a}=$ zero vector , may be not satisfied in the case of the mass vector. Also, according to the confined theory, the measured mass $m$ of the composite particle of $N$ constituents $m_{i}, i=1,2, \ldots . ., N$, in some cases, is greater than the sum $\sum_{i=1}^{N} m_{i}$. For example, ${ }^{\Delta}$-particle and $\rho$-meson. This means that, in some cases, the triangle inequality of the mathematical norm is not satisfied in the case of masses of particles, i. e., in some cases we have $m \geq \sum_{i=1}^{N} m_{i}$. Therefore, since the mass vector is defined such that

$$
\text { (the magnitude of the mass vector })^{2}=m \text {, }
$$

we will consider the following definition for the norm $\|\mathbf{m}\|_{\text {of a mass vector }} \mathbf{m}$

$$
\|\mathbf{m}\|=(\mathbf{m} \circ \mathbf{m})^{1 / 2}=\left(m_{t}-W\right)^{1 / 2}=\sqrt{m} .
$$

It is clear that $\|\mathbf{m}\| \geq 0$.

Now, we consider two cases of a mass vector $\mathbf{m}=\mathbf{m}_{1}+\mathbf{m}_{2}$. The first case where $m<m_{1}+m_{2}$. In this case we get 
$\|\mathbf{m}\|^{2}<\left\|\mathbf{m}_{1}\right\|^{2}+\left\|\mathbf{m}_{2}\right\|^{2}<\left\|\mathbf{m}_{1}\right\|^{2}+\left\|\mathbf{m}_{2}\right\|^{2}+2\left\|\mathbf{m}_{1}\right\|\left\|\mathbf{m}_{2}\right\|$ $=\left(\left\|\mathbf{m}_{1}\right\|+\left\|\mathbf{m}_{2}\right\|\right)^{2}$,

then,

$$
\left\|\mathbf{m}_{1}+\mathbf{m}_{2}\right\|<\left\|\mathbf{m}_{1}\right\|+\left\|\mathbf{m}_{2}\right\|
$$

In the second case we have $m>m_{1}+m_{2}$, therefore,

$$
\begin{aligned}
\|\mathbf{m}\|^{2} & >\left\|\mathbf{m}_{1}\right\|^{2}+\left\|\mathbf{m}_{2}\right\|^{2} \\
& >\left\|\mathbf{m}_{1}\right\|^{2}+\left\|\mathbf{m}_{2}\right\|^{2}-2\left\|\mathbf{m}_{1}\right\|\left\|\mathbf{m}_{2}\right\|, \\
& =\left(\left\|\mathbf{m}_{1}\right\|-\left\|\mathbf{m}_{2}\right\|\right)^{2}
\end{aligned}
$$

then,

$$
\left\|\mathbf{m}_{1}+\mathbf{m}_{2}\right\|>\left\|\mathbf{m}_{1}\right\|-\left\|\mathbf{m}_{2}\right\|
$$

\subsubsection{Zero-norm Mass Vector}

Consider a particle of measured mass equals zero, like photon. Let $\mathbf{m}=\left(\sqrt{m_{t}}, \mathbf{W}\right)$ is the mass vector of this particle. Then,

$$
\|\mathbf{m}\|^{2}=\mathbf{m} \circ \mathbf{m}=m_{t}-W=m=0
$$

At the same time, this particle has a field with virtual mass $W$. Thus, from (24), the total mass of the particle is

$$
m_{t}=m+W=0+W=W \neq 0
$$

and we can write the mass vector of zero measured mass particle in the form

$$
\mathbf{m}=(\sqrt{W}, \mathbf{W})
$$

Note that, $\|\mathbf{m}\|=0$, at the same time $\mathbf{m} \neq$ zero vector. The mass vectors of zero-norm correspond to the light cone in the time-space reference frame where the motion of the zero measured mass particles is considered.

The measured mass for a field can be considered equals zero. Therefore, if the virtual mass of the field is equal ${ }_{\text {to }} W_{f}$, we can define the mass vector of the field in the form

$$
\mathbf{m}_{f}=\left(\sqrt{W_{f}}, \mathbf{W}_{f}\right)
$$

where $\mathbf{W}_{f}$ is a three-dimensional vector with (as a definition) $\left\|\mathbf{W}_{f}\right\|^{2}=\mathbf{W}_{f} \bullet \mathbf{W}_{f}=W_{f}$.

Denoting to the mass vector of the antiparticle of a particle $^{\mathbf{m}}$ by $\tilde{\mathbf{m}}$, we have $\mathbf{m}+\tilde{\mathbf{m}}=\mathbf{g}$, where the mass vector $\mathbf{g}$ represents a massless particle.

\subsection{A Particle in a Field}

Consider a particle of the mass vector $\mathbf{m}=\left(\sqrt{m_{t}}, \mathbf{W}\right)$ in a field of the mass vector $\mathbf{m}_{f}=\left(\sqrt{W_{f}}, \mathbf{W}_{f}\right)$, where the particle and the field have the same kind of force. Thus, if we consider the particle in the field as a new system, this system has a new mass vector ${ }^{f}$ which is given by

$$
\mathbf{m}^{f}=\mathbf{m}+\mathbf{m}_{f}=\left(\sqrt{m_{t}+W_{f}}, \mathbf{W}+\mathbf{W}_{f}\right) .
$$

Then,

$$
\begin{aligned}
m^{f} & =\left\|\mathbf{m}^{f}\right\|^{2} \\
& =\left(\sqrt{m_{t}+W_{f}}, \mathbf{W}+\mathbf{W}_{f}\right) \circ\left(\sqrt{m_{t}+W_{f}}, \mathbf{W}+\mathbf{W}_{f}\right) \\
& =m_{t}+W_{f}-W-W_{f}-\mathbf{W} \bullet \mathbf{W}_{f}-\mathbf{W}_{f} \bullet \mathbf{W} \\
& =m-2 \lambda_{p f} \sqrt{W} \sqrt{W_{f}},
\end{aligned}
$$

where we can consider $\mathbf{W} \bullet \mathbf{W}_{f}=\mathbf{W}_{f} \bullet \mathbf{W}$ and $\lambda_{p f}$ is the interaction parameter of the particle with the field. It is clear that, even with $\left\|\mathbf{m}_{f}\right\|=0$, the inner product $\mathbf{m} \circ \mathbf{m}_{f}$, as defined by equation (12), might not be equal zero. The quantity $2 \lambda_{p f} \sqrt{W} \sqrt{W_{f}}$ represents the interaction energy between the particle and the field as it is expected. $\lambda_{p f}=0$ represents the free space (no field) and we have $m^{f}=m$. If $\lambda_{p f}>0$ the quantity $2 \lambda_{p f} \sqrt{W} \sqrt{W_{f}}$ is positive and $m^{f}<m$. This means that the field in this case is attractive field and the quantity $2 \lambda_{p f} \sqrt{W} \sqrt{W_{f}}$ represents the binding energy of the particle in the field. In the case $\lambda_{p f}<0$ the quantity $2 \lambda_{p f} \sqrt{W} \sqrt{W_{f}}$ is negative and $m^{f}>m$. Therefore, the field in this case can be considered a repulsive field and the quantity $2 \lambda_{p f} \sqrt{W} \sqrt{W_{f}}$ represents the gained energy of the particle from the field. From equation (29) and the above three cases we can say that $m^{f}$ is the mass of the particle in the field. If the field is attractive the particle mass decreases and if the field is repulsive the particle mass increases. Thus, the particle mass changes according to two factors. The first is the speed ${ }^{v}$ of the particle according to the theory of relativity and the second is the surrounding space of the particle, is it free or not. Therefore, we must introduce the concept of the rest mass of the particle in the field $m_{0}^{f}$, which is different from the mass of the particle in the free space at rest $m_{0}$. Thus, in the study of the motion of a relativistic particle in a field, for the mass of the particle we must use the rule $m^{f}=m_{0}^{f} / \sqrt{1-(v / c)^{2}}$, where $m_{0}^{f}$ is the mass of the particle in the field at rest. For example, in the nonrelativistic case, the kinetic energy of the free motion is $\frac{1}{2} m_{0} v^{2}$ while in the field is $\frac{1}{2} m_{0}^{f} v^{2}$. It is clear that $m_{0}^{f}=m_{0}-2 \mathbf{W} \bullet \mathbf{W}_{f} \cdot$ This means that for free massless particle, where $m_{0}=0$, the rest mass of this particle in the field equals to $m_{0}^{f}=-2 \mathbf{W} \bullet \mathbf{W}_{f} \neq 0$. This result is consistent with the existence of the Higgs field through the space and it cause existing particles to acquire mass instead of new massless particles being formed [5]. 


\section{The General Mass Space}

Most of the particles have the four kinds of force. Therefore, we need a general mass space $M$ consists of the general mass vectors of the particles. This general mass vector of a particle must represent, simultaneously, the different forces which the particle has. At the same time, we cannot use the sum of the mass vectors $\mathbf{m}_{s}, \mathbf{m}_{e}$, $\mathbf{m}_{g}$ and $\mathbf{m}_{w}$ to obtain the general mass vector $\mathbf{m}_{G}$. This is because each of the mass vectors $\mathbf{m}_{s}, \mathbf{m}_{e}, \mathbf{m}_{g}$ and $\mathbf{m}_{w}$ has different charge axes. These axes are not arbitrary and are specified for each kind of force. Thus, we can suggest $\mathbf{m}_{G}$ in the form

$$
\mathbf{m}_{G}=\left(\sqrt{m_{G t}}, \mathbf{w}_{s}, \mathbf{W}_{e}, \mathbf{w}_{g}, \mathbf{W}_{w}\right) .
$$

The order of the vectors $\mathbf{W}_{s}, \mathbf{W}_{e}, \mathbf{W}_{g}$ and $\mathbf{W}_{w}$ in this form is not important. Note that $m_{G}=m$. Thus, in general, the general mass vector $\mathbf{m}_{G}$ has 13 dimensions and each mass space of the four mass spaces $M_{s}, M_{e}$, $M_{g}$ and $M_{w}$ is a subspace of the general mass space $\mathrm{M}$. For a general mass vector $\mathbf{m}_{G}$ we have

$$
\begin{aligned}
m & =\mathbf{m}_{G} \circ \mathbf{m}_{G} \\
& =m_{G t}-\mathbf{W}_{s} \bullet \mathbf{W}_{s}-\mathbf{W}_{e} \bullet \mathbf{W}_{e}-\mathbf{W}_{g} \bullet \mathbf{W}_{g}-\mathbf{W}_{w} \bullet \mathbf{W}_{w} \\
& =m_{G t}-W_{s}-W_{e}-W_{g}-W_{w},
\end{aligned}
$$

Then,

$$
m_{G t}=m+W_{s}+W_{e}+W_{g}+W_{w}
$$

Thus, the total mass in equation (30) equals the measured mass of the particle plus the virtual masses of the four fields. This equation can be considered as a mass equation of the particle. The total mass $m_{G t}$ in this equation must be the actual total mass.

For a particle in, for example, an electromagnetic field, if we use the general mass vector of the particle $\mathbf{m}_{G}=\left(\sqrt{m_{G t}}, \mathbf{W}_{s}, \mathbf{W}_{e}, \mathbf{W}_{g}, \mathbf{W}_{w}\right)$ and the mass vector of the field $\mathbf{m}_{f}=\left(\sqrt{W_{e f}}, \mathbf{W}_{e f}\right)$ we have the same result as (29). This because, in the general mass space the vector $\mathbf{m}_{f}=\left(\sqrt{W_{e f}}, \mathbf{W}_{e f}\right)$ can be considered as a vector in the general mass space and can be written it as $\mathbf{m}_{f}=\left(\sqrt{W_{e f}}, 0, \mathbf{W}_{e f}, 0,0\right)$.

\subsection{The General Mass Equation}

In the general case of $n$-constituent particle of the measured mass $m$ and the general mass vector $\mathbf{m}_{G}$, the mass equation of the this composite particle, from equations (8) and (12), is given by

$$
\begin{aligned}
m & =\left(\left(\sum_{i=1}^{n} m_{i G t}\right)^{1 / 2}, \sum_{i=1}^{n} \mathbf{W}_{i g}, \sum_{i=1}^{n} \mathbf{W}_{i e}, \sum_{i=1}^{n} \mathbf{W}_{i s}, \sum_{i=1}^{n} \mathbf{W}_{i w}\right) \\
& \circ\left(\left(\sum_{j=1}^{n} m_{j G t}\right)^{1 / 2}, \sum_{j=1}^{n} \mathbf{W}_{j g}, \sum_{j=1}^{n} \mathbf{W}_{j e}, \sum_{j=1}^{n} \mathbf{W}_{j s}, \sum_{j=1}^{n} \mathbf{W}_{j w}\right) \\
= & \sum_{i=1}^{n}\left(m_{i G t}-W_{i g}-W_{i e}-W_{i s}-W_{i w}\right)-\sum_{i \neq j}^{n} \mathbf{W}_{i g} \bullet \mathbf{W}_{j g} \\
& -\sum_{i \neq j}^{n} \mathbf{W}_{i e} \bullet \mathbf{W}_{j e}-\sum_{i \neq j}^{n} \mathbf{W}_{i s} \bullet \mathbf{W}_{j s}-\sum_{i \neq j}^{n} \mathbf{W}_{i w} \bullet \mathbf{W}_{j w} .
\end{aligned}
$$

Therefore,

$$
\begin{aligned}
& m=\sum_{i=1}^{n} m_{i}-\sum_{i \neq j}^{n} \mathbf{W}_{i g} \bullet \mathbf{W}_{j g}-\sum_{i \neq j}^{n} \mathbf{W}_{i e} \bullet \mathbf{W}_{j e} \\
& -\sum_{i \neq j}^{n} \mathbf{W}_{i s} \bullet \mathbf{W}_{j s}-\sum_{i \neq j}^{n} \mathbf{W}_{i w} \bullet \mathbf{W}_{j w} .
\end{aligned}
$$

Then, the binding energy of $n$-constituent particle is given by

$$
\begin{aligned}
& E_{b}=m-\sum_{i=1}^{n} m_{i}=-2\left[\sum_{i<j}^{n} \mathbf{W}_{i g} \bullet \mathbf{W}_{j g}\right. \\
& \left.+\sum_{i<j}^{n} \mathbf{W}_{i e} \bullet \mathbf{W}_{j e}+\sum_{i<j}^{n} \mathbf{W}_{i s} \bullet \mathbf{W}_{j s}+\sum_{i<j}^{n} \mathbf{W}_{i w} \bullet \mathbf{W}_{j w}\right] .
\end{aligned}
$$

In equations (33) and (34), ${ }^{m}$ and $m_{i}$ are the measured masses of the composite particle and the constituent particles, respectively, which are invariant. In equation (34) we assumed that $\mathbf{W}_{i k} \bullet \mathbf{W}_{j k}=\mathbf{W}_{j k} \bullet \mathbf{W}_{i k}$ for any kind of force. If $E_{b}<0$ we have the usual case of composite particles and nuclei, and if $E_{b}>0$ we have the case of resonances where the confined theory is satisfied. Equation (33) can be considered as a general mass equation where the role of each kind of force is considered.

\section{Applications}

We shall present some applications of the general mass equation (33) and we try to obtain some physical results and to prove that the suggested physical meaning of the terms $\mathbf{W}_{i k} \bullet \mathbf{W}_{j k}$ as the energy of two particles interaction is true. Some of these applications were given before in the work of Hassan [1]. But, in the present work we use the second definition of the two mass vectors addition. In these applications a mass parabola equation for nuclei is obtained in terms of the nucleon-nucleon interactions. Comparing our mass equation with the semiempirical Weizsäcker mass formula some well-known properties of nucleon-nucleon interaction can be observed. Also, the values of neutron-proton, proton-proton and neutron-neutron strong interaction energy ( $\mathbf{W}_{n s} \bullet \mathbf{W}_{p s}$, $\mathbf{W}_{p s} \bullet \mathbf{W}_{p^{\prime} s}$ and $\mathbf{W}_{n s} \bullet \mathbf{W}_{n^{\prime} s}$, respectively) are calculated. The proton-proton Coulomb energy

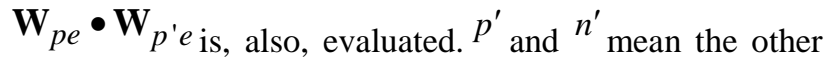
proton and neutron, respectively.

\subsection{A Mass Parabola Equation for Nuclei}


For a nucleus of mass number $A$ and atomic number $Z$, using equation (33), we have

$$
\begin{aligned}
& m=\sum_{i=1}^{A} m_{i}-2\left[\sum_{i<j}^{A} \mathbf{W}_{i g} \bullet \mathbf{W}_{j g}+\sum_{i<j}^{A} \mathbf{W}_{i e} \bullet \mathbf{W}_{j e}\right. \\
& \left.+\sum_{i<j}^{A} \mathbf{W}_{i s} \bullet \mathbf{W}_{j s}+\sum_{i<j}^{A} \mathbf{W}_{i w} \bullet \mathbf{W}_{j w}\right] .
\end{aligned}
$$

Since neutron-proton and neutron-neutron electromagnetic interactions can be considered nonexistent, we can take $\mathbf{W}_{i e} \bullet \mathbf{W}_{j e}=0$ for $i=n, p$ and $j$ $=n$. The gravitational and weak interactions between the nucleons are very small, specially, with respect to the electromagnetic and strong interactions. Therefore, as an approximation, we can neglect the terms contain $\mathbf{W}_{i g} \bullet \mathbf{W}_{j g}$ and $\mathbf{W}_{i w} \bullet \mathbf{W}_{j w}$ for $i \neq j$. Also, we can consider, as an approximation, that $\quad \mathbf{W}_{i e} \bullet \mathbf{W}_{j e}=\mathbf{W}_{p e} \bullet \mathbf{W}_{p^{\prime} e}$ $\mathbf{W}_{i s} \bullet \mathbf{W}_{j s}=\mathbf{W}_{p s} \bullet \mathbf{W}_{p^{\prime} s}, \quad \mathbf{W}_{i s} \bullet \mathbf{W}_{j s}=\mathbf{W}_{n s} \bullet \mathbf{W}_{n^{\prime} s}$ and $\mathbf{W}_{i s} \bullet \mathbf{W}_{j s}=\mathbf{W}_{n s} \bullet \mathbf{W}_{p s}$ for any two different protons, any two different neutrons and any neutronproton pair, respectively. For the same proton (neutron) $\mathbf{W}_{p s} \bullet \mathbf{W}_{p s}=W_{p s} \quad\left(\mathbf{W}_{n s} \bullet \mathbf{W}_{n s}=W_{n s}\right) \quad$ which represents the energy of the proton (neutron) field. But, for two different protons (neutrons) the quantity $\mathbf{W}_{p s} \bullet \mathbf{W}_{p^{\prime} s}\left(\mathbf{W}_{n s} \bullet \mathbf{W}_{n^{\prime} s}\right.$ ) is related to the states of each proton (neutron) and represents the energy of the interaction between two different particles. Therefore, we cannot assume that $\mathbf{W}_{p s} \bullet \mathbf{W}_{p^{\prime} s}=\mathbf{W}_{p s} \bullet \mathbf{W}_{p s}=W_{p s}$, or $\quad \mathbf{W}_{n s} \bullet \mathbf{W}_{n^{\prime} s}=\mathbf{W}_{n s} \bullet \mathbf{W}_{n s}=W_{n s} \quad$. Taking $i, j=1,2, \ldots . Z$ for protons and $i, j=Z+1, Z+2, \ldots . A$ for neutrons, equation (35) can be written as

$$
\begin{aligned}
m & =\left[2 \mathbf{W}_{n s} \bullet \mathbf{W}_{p s}-\mathbf{W}_{p e} \bullet \mathbf{W}_{p^{\prime} e}\right. \\
& \left.-\mathbf{W}_{p s} \bullet \mathbf{W}_{p^{\prime} s}-\mathbf{W}_{n s} \bullet \mathbf{W}_{n^{\prime} s}\right] Z^{2} \\
& +\left[m_{p}-m_{n}+\mathbf{W}_{p e} \bullet \mathbf{W}_{p^{\prime} e}+\mathbf{W}_{p s} \bullet \mathbf{W}_{p^{\prime} s}\right. \\
& \left.+(2 A-1) \mathbf{W}_{n s} \bullet \mathbf{W}_{n^{\prime} s}-2 A \mathbf{W}_{n s} \bullet \mathbf{W}_{p s}\right] Z \\
& +\left[m_{n}-(A-1) \mathbf{W}_{n s} \bullet \mathbf{W}_{n^{\prime} s}\right] A .
\end{aligned}
$$

Equation (36) represents a mass parabola equation similar to the semi-empirical Weizsäcker mass formula [3]

$$
m=\gamma Z^{2}+\beta Z+\alpha A
$$

where

$$
\begin{gathered}
\gamma=\frac{4 C_{a s y}}{A}+\frac{C_{c o u l}}{A^{1 / 3}} \\
\beta=m_{p}-m_{n}-4 C_{a s y}-\frac{C_{c o u l}}{A^{1 / 3}} \\
\alpha=m_{n}-C_{v o l}+C_{a s y}+\frac{C_{\text {sur }}}{A^{1 / 3}} \mp \frac{C_{\text {pair }}}{A^{7 / 4}}(\text { or zero }),(38 \mathrm{c})
\end{gathered}
$$

and the coefficients $C_{\text {vol }}, C_{\text {sur }}, C_{a s y}, C_{\text {coul }}$ and $C_{\text {pair }}$ are related to volume-, surface-, asymmetry-, Coulomb- and pairing-energy. The last term in equation (38c) equals zero for odd A. The negative and positive sign for even A correspond to even- and odd-Z, respectively. In the coulomb-energy term $-C_{\text {coul }} / A^{1 / 3}$, the spherical uniform charge distribution for nuclei is assumed. Comparing equations (36) and (37), using (38), we get

$$
\begin{gathered}
\left(4 C_{a s y} / A\right)+\frac{C_{c o u l}}{A^{1 / 3}}\left(C_{\text {coul }} / A^{1 / 3}\right) \\
=2 \mathbf{W}_{n s} \bullet \mathbf{W}_{p s}-\mathbf{W}_{p e} \bullet \mathbf{W}_{p^{\prime} e} \\
-\mathbf{W}_{p s} \bullet \mathbf{W}_{p^{\prime} s}-\mathbf{W}_{n s} \bullet \mathbf{W}_{n^{\prime} s}, \\
-4 C_{a s y}-\frac{C_{c o u l}}{A^{1 / 3}}=\mathbf{W}_{p e} \bullet \mathbf{W}_{p^{\prime} e}+\mathbf{W}_{p s} \bullet \mathbf{W}_{p^{\prime} s} \\
+(2 A-1) \mathbf{W}_{n s} \bullet \mathbf{W}_{n^{\prime} s}-2 A \mathbf{W}_{n s} \bullet \mathbf{W}_{p s}, \\
-C_{v o l}+C_{a s y}+\frac{C_{s u r}}{A^{1 / 3}} \mp \frac{C_{p a i r}}{A^{7 / 4}}(\text { or } 0)=-(A-1) \mathbf{W}_{n s} \bullet \mathbf{W}_{n^{\prime} s}
\end{gathered}
$$

Solving these equations, (39), with respect to $\mathbf{W}_{n s} \bullet \mathbf{W}_{n^{\prime} s} \quad, \quad \mathbf{W}_{p e} \bullet \mathbf{W}_{p^{\prime} e}+\mathbf{W}_{p s} \bullet \mathbf{W}_{p^{\prime} s} \quad$ and $\mathbf{W}_{n s} \bullet \mathbf{W}_{p s}$ we have

$$
\begin{gathered}
\mathbf{W}_{n s} \bullet \mathbf{W}_{n^{\prime} s}=\frac{1}{A-1}\left[C_{v o l}-C_{a s y}-\frac{C_{\text {sur }}}{A^{1 / 3}} \pm \frac{C_{\text {pair }}}{A^{7 / 4}}(\text { or } 0)\right],(40 \mathrm{a}) \\
\mathbf{W}_{p e} \bullet \mathbf{W}_{p^{\prime} e}+\mathbf{W}_{p s} \bullet \mathbf{W}_{p^{\prime} s}=\frac{1}{A-1}\left[C_{v o l}-C_{a s y}(40 \mathrm{~b})\right. \\
\left.-\frac{C_{\text {sur }}}{A^{1 / 3}}-\frac{(A-1) C_{\text {coul }}}{A^{1 / 3}} \pm \frac{C_{p a i r}}{A^{7 / 4}}(\text { or } 0)\right], \\
\mathbf{W}_{n s} \bullet \mathbf{W}_{p s}=\frac{1}{A-1}\left[C_{v o l}+\frac{A-2}{A} C_{a s y}\right. \\
\left.-\frac{C_{s u r}}{A^{1 / 3}} \pm \frac{C_{p a i r}}{A^{7 / 4}}(\text { or } 0)\right] .
\end{gathered}
$$

The similarity among (40a), (40b) and (40c) is clear and represents the isospin invariant of nuclear force in Weizsäcker mass formula. The little difference between them can be understood as a result of the electric force and asymmetry effect. The difference between $\mathbf{W}_{n s} \bullet \mathbf{W}_{n ' s}$ and $\mathbf{W}_{p e} \bullet \mathbf{W}_{p^{\prime} e}+\mathbf{W}_{p s} \bullet \mathbf{W}_{p^{\prime} s}$ is the electric force term, $\mathbf{W}_{p e} \bullet \mathbf{W}_{p^{\prime} e}=-\frac{C_{\text {coul }}}{A^{1 / 3}}$

Therefore,

we have $\mathbf{W}_{p s} \bullet \mathbf{W}_{p^{\prime} s}=\mathbf{W}_{n s} \bullet \mathbf{W}_{n^{\prime} s}$. The negative sign in $\mathbf{W}_{p e} \bullet \mathbf{W}_{p^{\prime} e}$ due to that this quantity represents a repulsive force in nuclei. Also, the difference between $\mathbf{W}_{p s} \bullet \mathbf{W}_{p^{\prime} s}$ and $\mathbf{W}_{n s} \bullet \mathbf{W}_{n^{\prime} s}$ on one side and $\mathbf{W}_{n s} \bullet \mathbf{W}_{p s}$ on the other side is represented by the asymmetry term, as we must have expected.

For nuclei with $A \geq 4$, where we can use the Weizsäcker mass formula, the virtual masses (energies) of the electromagnetic interaction $\mathbf{W}_{p e} \bullet \mathbf{W}_{p^{\prime} e}$, the strong proton-proton interaction $\mathbf{W}_{p s} \bullet \mathbf{W}_{p^{\prime} s}$, the strong neutron-neutron interaction $\mathbf{W}_{n s} \bullet \mathbf{W}_{n ' s}$ and the strong neutron-proton interaction $\mathbf{W}_{n s} \bullet \mathbf{W}_{p s}$ can be calculated 
using the above equations. The results are given in Table 1 for many nuclei, where $C_{v o l}=16.11 \mathrm{MeV}$ $C_{\text {sur }}=20.21 \mathrm{MeV} \quad, \quad C_{\text {asy }}=20.65 \mathrm{MeV}$ $C_{\text {coul }}=0.583 \mathrm{MeV}$ and $C_{\text {pair }}=33 \mathrm{MeV}$ are used [3]. It is clear that, from Table $1, \mathbf{W}_{p s} \bullet \mathbf{W}_{p^{\prime} s}<0$ and $\mathbf{W}_{n s} \bullet \mathbf{W}_{n ' s}<0$ for all nuclei. This means that the contributions of proton-proton and neutron-neutron interactions in the binding energy of a nucleus lead to its disconnection, i.e., these interaction energies, $\mathbf{W}_{p s} \bullet \mathbf{W}_{p ' s}$ and $\mathbf{W}_{n s} \bullet \mathbf{W}_{n^{\prime} s}$, represent a repulsive forces. At the same time the holding of the nucleus is due to neutron-proton interaction where $\mathbf{W}_{n s} \bullet \mathbf{W}_{p s}>0$. We must note that, for all nuclei, except ${ }^{6} \mathrm{Li}_{3}$, in Table 1 we have

$\left|\mathbf{W}_{p s} \bullet \mathbf{W}_{p^{\prime} s}\left(\operatorname{or} \mathbf{W}_{n s} \bullet \mathbf{W}_{n^{\prime} s}\right)+\mathbf{W}_{p e} \bullet \mathbf{W}_{p^{\prime} e}\right|<\mathbf{W}_{n s} \bullet \mathbf{W}_{p s}$

. For ${ }^{6} \mathrm{Li}_{3}$ both sides of inequality, approximately, are the same. These results interpret the absence of proton-proton and neutron-neutron bound system and the existence of the deuteron. It is a generalization of the rule of the interaction of similar and different electric charges. The force between two

\begin{tabular}{|c|c|c|c|}
\hline Nucleus & $\mathbf{W}_{p s} \bullet \mathbf{W}_{p^{\prime} s}=\mathbf{W}_{n s} \bullet \mathbf{W}_{n^{\prime} s}, \mathrm{MeV}$ & $\mathbf{W}_{n s} \bullet \mathbf{W}_{p s}$ & $\mathbf{W}_{p e} \bullet \mathbf{W}_{p^{\prime} e}$ \\
\hline${ }^{4} \mathrm{He}_{2}$ & -4.7849 & 5.5401 & -0.3673 \\
\hline${ }^{6} \mathrm{Li}_{3}$ & -3.4193 & 3.4640 & -0.3208 \\
\hline${ }^{7} \mathrm{Li}_{3}$ & -2.5175 & 3.3825 & -0.3048 \\
\hline${ }^{9} \mathrm{Be}_{4}$ & -1.7820 & 2.8069 & -0.2803 \\
\hline${ }^{12} C_{6}$ & -1.1765 & 2.2652 & -0.2546 \\
\hline${ }^{14} N_{7}$ & - 1.0193 & 1.9307 & -0.2419 \\
\hline${ }^{15} N_{7}$ & -0.9096 & 1.8437 & -0.2364 \\
\hline${ }^{40} \mathrm{Ca}_{20}$ & -0.2666 & 0.7659 & -0.1705 \\
\hline${ }^{41} \mathrm{Ca}_{20}$ & -0.2600 & 0.7473 & -0.1691 \\
\hline${ }^{50} V_{23}$ & -0.2053 & 0.6207 & -0.1583 \\
\hline${ }^{117} \mathrm{Te}_{52}$ & -0.0748 & 0.2782 & -0.1192 \\
\hline${ }^{202} \mathrm{Hg}_{80}$ & -0.0397 & 0.1647 & -0.0994 \\
\hline${ }^{203} \mathrm{Hg}_{80}$ & -0.0395 & 0.1639 & -0.0992 \\
\hline${ }^{207} \mathrm{~Pb}_{82}$ & -0.0386 & 0.1609 & -0.0986 \\
\hline
\end{tabular}

protons or two neutrons is repulsive and the force between proton and neutron is attractive. Also, it is interpret the hard core of some suggested nuclear potentials.

From Table 1, we can, also, see that the absolute value of the energy of any nucleon-nucleon interaction $\mathbf{W}_{i k} \bullet \mathbf{W}_{j k}, i, j=p, n ; k=e, s_{\text {decreases as the mass }}$ number $A$ increases. This, may be due to the finite value of the virtual mass of any force for any particle. Therefore, the contribution of any particle in nucleon-nucleon interaction energy decreases as the mass number $A$ increases.

It is important to note that the isospin invariant result $\mathbf{W}_{p s} \bullet \mathbf{W}_{p^{\prime} s}=\mathbf{W}_{n s} \bullet \mathbf{W}_{n^{\prime} s}$ is obtained under many approximations. Also, we compare our mass equation with the Weizsäcker mass formula where the isospin invariant is considered. Therefore, in general, we must write $\mathbf{W}_{p s} \bullet \mathbf{W}_{p^{\prime} s} \approx \mathbf{W}_{n s} \bullet \mathbf{W}_{n^{\prime} s}$, i.e., the isospin invariant is an approximation. This means the violation of the charge symmetry [6], i.e., the charge symmetry is an approximation.

\subsection{The Mass Equation of Deuteron}

Consider now the case of the simplest complex nucleus, deuteron, where $A=2$ and $Z=1$. For this case the Weizsäcker mass formula does not apply. But, using equation (36) we can obtain the mass equation of deuteron. Using this mass equation we try to obtain some wellknown information about the energy of the neutron-proton interaction in this nucleus. For the deuteron, the mass equation (36) takes the form

$$
m=m_{p}+m_{n}-2\left(\mathbf{W}_{n s} \bullet \mathbf{W}_{p s}\right)
$$

Thus, the binding energy of the deuteron is

$$
E_{b}=m-\left(m_{p}+m_{n}\right)=-2\left(\mathbf{W}_{n s} \bullet \mathbf{W}_{p s}\right) .
$$

Using the well-known value of the deuteron binding energy we get

$$
-2.226=-2\left(\mathbf{W}_{n s} \bullet \mathbf{W}_{p s}\right) .
$$

Then, in the deuteron 


$$
\mathbf{W}_{n s} \bullet \mathbf{W}_{p s}=1.113 \mathrm{MeV}>0
$$

Since, the total virtual energy of proton-neutron interaction, from equation (41), is $2\left(\mathbf{W}_{n s} \bullet \mathbf{W}_{p s}\right)$, we can assume that the contribution of each nucleon in the interaction is $\mathbf{W}_{n s} \bullet \mathbf{W}_{p s}=1.113 \mathrm{MeV}$, which is considered the binding energy per nucleon in the deuteron. Therefore, for the other nuclei the quantity $\mathbf{W}_{i k} \bullet \mathbf{W}_{j k}$ can be considered as a mean value of the contribution of the force $k$ in the binding energy of the nucleus per nucleon.

\subsection{The Mass Equations of Nuclei ${ }^{3} H_{1}$ and ${ }^{3} \mathrm{He}_{2}$}

In the case of the nucleus ${ }^{3} H_{1}$ the mass equation in our model, from equation (36), is

$$
\begin{aligned}
& \quad m=m_{p}+2 m_{n}-\left[2 \mathbf{W}_{n s} \bullet \mathbf{W}_{n^{\prime} s}+4 \mathbf{W}_{n s} \bullet \mathbf{W}_{p s}\right]( \\
& \text { For }{ }^{3} H e_{2} \text { we get } \\
& \qquad \begin{array}{l}
m=2 m_{p}+m_{n}-\left[2 \mathbf{W}_{p s} \bullet \mathbf{W}_{p^{\prime} s}\right. \\
\left.\quad+2 \mathbf{W}_{p e} \bullet \mathbf{W}_{p^{\prime} e}+4 \mathbf{W}_{n s} \bullet \mathbf{W}_{p s}\right]
\end{array}
\end{aligned}
$$

The binding energies of ${ }^{3} \mathrm{H}_{1}$ and ${ }^{3} \mathrm{He}_{2}$ are -8.48212 $\mathrm{MeV}$ and -7.71828 MeV, respectively. In these two nuclei we can consider the proton-neutron interaction $\quad \mathbf{W}_{n s} \bullet \mathbf{W}_{p s}$, proton-proton interactions $\mathbf{W}_{p s} \bullet \mathbf{W}_{p^{\prime} s}, \mathbf{W}_{p e} \bullet \mathbf{W}_{p^{\prime} e}$ and neutronneutron interaction $\mathbf{W}_{n s} \bullet \mathbf{W}_{n ' s}$ have, approximately, the same values in both nuclei. Thus, from equations (43) and (44), the difference between the binding energies of ${ }^{3} H_{1}$ and ${ }^{3} \mathrm{He}_{2}$ is -0.76384 and then

$$
\begin{aligned}
& \mathbf{W}_{p s} \bullet \mathbf{W}_{p^{\prime} s}+\mathbf{W}_{p e} \bullet \mathbf{W}_{p^{\prime} e} \\
& -\mathbf{W}_{n s} \bullet \mathbf{W}_{n^{\prime} s}=-0.38192
\end{aligned}
$$

$$
\begin{gathered}
\text { Note that, if } \begin{array}{l}
\text { we } \\
\mathbf{W}_{p e} \bullet \mathbf{W}_{p^{\prime} e}
\end{array}=-C_{\text {coul }} / A^{1 / 3} \\
C_{\text {coul }}=0.55083 \mathrm{MeV} \text {, for }{ }^{3} \mathrm{He}_{2} \text {, we get, } \\
\text { using } \\
\mathbf{W}_{p e} \bullet \mathbf{W}_{p^{\prime} e}=-0.38192 \text {.Thus, } \\
\mathbf{W}_{p s} \bullet \mathbf{W}_{p^{\prime} s}-\mathbf{W}_{n s} \bullet \mathbf{W}_{n^{\prime} s}=0
\end{gathered}
$$

which agrees with the isospin invariant of nuclear force under the considered approximations. From this result we can conclude that the difference between the binding energies of the two mirror nuclei ${ }^{3} \mathrm{H}_{1}$ and ${ }^{3} \mathrm{He}_{2}$ due, only, to the Coulomb energy.

\subsection{A Relation of $\mathbf{W}_{i k} \bullet W_{i^{\prime} k}$ with the Usual Charge and Potential}

In the case of a nucleus the quantity $\mathbf{W}_{p e} \bullet \mathbf{W}_{p^{\prime} e}$, for example, can be calculated using the Coulomb potential and a suitable charge distribution for the nucleus. The Coulomb energy of a charge distribution described by a density $\rho$ that is spatially bounded and everywhere finite is given by [7]

$$
\mathrm{W}_{\text {coul }}=\frac{1}{2} \int d \mathbf{r} \rho(r) \phi(r),
$$

where $\phi(r)$ is the potential and the volume integral is over the region enclosing all the charge. For a nucleus of atomic number $Z$ the Coulomb energy of proton-proton pair is

$$
E_{\text {coul }}=\mathrm{W}_{\text {coul }} /[Z(Z-1) / 2]=(1 / Z(Z-1)) \int d \mathbf{r} \rho(r) \phi(r)
$$

For a sphere of uniform charge density and radius $R$ the Coulomb energy for $Z(Z-1)$ / 2 proton-proton pairs, using (47), is

$$
\mathrm{W}_{\text {coul }}=\frac{3}{5} \frac{Z(Z-1) e^{2}}{5 \pi \varepsilon_{0} R}
$$

where $e^{e}$ is the proton charge and $\varepsilon_{0}$ is the vacuum permittivity. Assuming $R=r_{0} A^{1 / 3}$, this expression takes the form

$$
\mathrm{W}_{\text {coul }}=C_{\text {coul }} Z(Z-1) A^{-1 / 3}
$$

and the Coulomb energy for one proton-proton pair is given by

$$
E_{\text {coul }}=\mathrm{W}_{\text {coul }} /[Z(Z-1) / 2]=2 C_{\text {coul }} A^{-1 / 3}
$$

Thus, the Coulomb energy per proton in one protonproton pair is $E_{\text {coul }} / 2$. But, from equations (40b) and (40c), the Coulomb energy per proton in one protonproton pair $\mathbf{W}_{p e} \bullet \mathbf{W}_{p^{\prime} e}=-\frac{C_{\text {coul }}}{A^{1 / 3}}$. Then, $\mathbf{W}_{p e} \bullet \mathbf{W}_{p^{\prime} e}=-\frac{1}{2} E_{\text {coul }}$. Therefore, we can write

$$
\mathbf{W}_{p e} \bullet \mathbf{W}_{p^{\prime} e}=-\frac{1}{2 Z(Z-1)} \int d \mathbf{r} \rho_{e}(r) \phi_{e}(r)
$$

where $\rho_{e}(r)$ and $\phi_{e}(r)$ are the electric charge density and the Coulomb potential in the nucleus, respectively.

Now, for any kind of force we can, similarly, suggest

$$
\begin{aligned}
& \mathbf{W}_{i k} \bullet \mathbf{W}_{i^{\prime} k}=-\frac{1}{2 N_{\text {pair }}} \int d \mathbf{r} \rho_{k}(r) \phi_{k}(r), \\
& k=e, g, s, w ; i, i^{\prime}=p, n
\end{aligned}
$$

where $N_{\text {pair }}$ is the number of pairs in each case, $\rho_{g}$, $\rho_{s}, \rho_{w}$ and $\phi_{g}, \phi_{s}, \phi_{w}$ are the gravitational, strong and weak charge densities and potentials, respectively.

\section{Conclusions}

In conclusion, this article can be considered as methodical work in physics using a mathematical point of view. I know that the suggested concept of mass vector is 
unusual concept, but, using it we obtained the following: 1) A general definition of charge of any kind of force as a configuration of energy. 2) A general mass equation of any elementary or composite particle in terms of all kinds of force, instead of putting the different terms by hand. Also, in the framework of the mass equation the confined theory can be understood. 3) Using the mass vector of a field and the mass vector of a massless particle we can proved, mathematically, that the massless particle in a field has a measured mass. Therefore, we can answer on the question, why the light has gravitational interaction? At the same time this is, also, a mathematical proof for the existence of Higgs boson. Also, using the same result of a particle in a field we can introduced the concepts of free rest mass of the particle and the rest mass in a field. 4) With the given applications of the mass equation in the case of nuclei we can decide that the proton-proton and neutron-neutron strong interactions represent repulsive forces and only the neutron-proton interaction represents attractive force. This interpret the absence of protonproton and neutron-neutron bound states. Only the neutron-proton bound state, deuteron, was found. Also, these results are consistent with the general sense of the interaction between two similar things. The presence of nuclei is not given sufficient justification for considering that between protons attractive forces, as well as between neutrons, as long as the force between the proton- neutron pair is attractive and larger than the force between any pair of protons or neutrons.

\section{References}

[1] Hassan, M. A., "A vector analog for the mass- Model and predictions,” Proc INSA, A63 (6). 437-456. Jul. 1997.

[2] Thomson, J. J., "On the Electric and Magnetic Effects produced by the Motion of Electrified Bodies," Philosophical Magazine, 5 11 (68). 229-249. 1881

[3] Waghmare, Y. R., Introductory Nuclear Physics, Oxford \&IBH publishing Co, New Delhi, 1981.

[4] Griffiths, D., Introduction to Elementary Particles, Harper \&Row, New York, 1987.

[5] ATLAS Collaboration, "Observation of a new particle in the search for the Standard Model Higgs boson with the ATLAS detector at the LHC”, Phys. Lett., B716 (1). 1-29. Sep. 2012.

[6] Biswas, S., Roy, P., and Dutt-Mazumder, A. K., " $\rho-\omega$ mixing and spin dependent CSV potential”, Indian J. Phys, 85(7). 1185-1189. Jul. 2011.

[7] Clemmow P. C., An Introduction To Electromagnetic Theory, Cambridge University Press, Cambridge, 1973. 